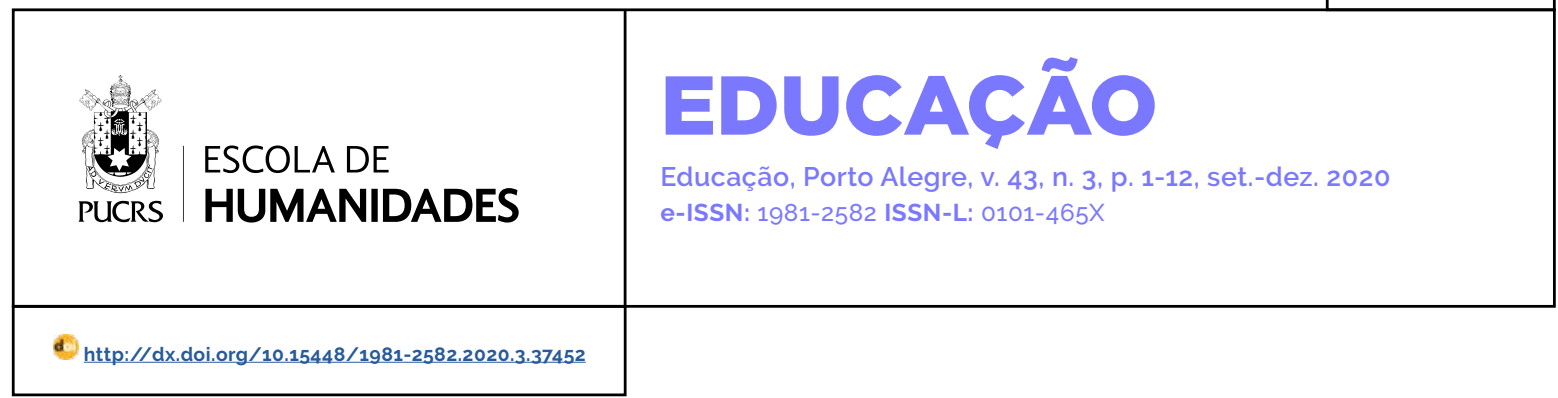

SEÇÃO: DOSSIÊ

\title{
O Estado da Arte ou o Estado do Conhecimento
}

The state of the art or the state of knowledge

El estado de arte o el estado del conocimiento

Anne Patricia Pimentel Nascimento da Silva ${ }^{1}$ orcid.org/0000-00018999-7799 annepp2000@yahoo.com.br

\section{Roberta Teixeira de Souza ${ }^{2}$ \\ orcid.org/0000-0002-5152-5048 rotsouza2@yahoo.com.br}

\section{Vera Maria Ramos de Vasconcellos ${ }^{1}$ orcid.org/0000-0001-9544-6600 vasconcellos.vera@gmail.com}

Recebido em: 26/6/2020 Aprovado em: 9/9/2020. Publicado em: 8/3/2021

\section{(C) (1)}

Artigo está licenciado sob forma de uma licença Creative Commons Atribuição 4.0 Internacional.
Resumo: O artigo inicia com uma discussão teórica do que são as revisões críticas dos modos de produção no campo da Educação reconhecidos como "Estado da Arte" e "Estado do Conhecimento". São estudos que sistematizam o que foi produzido durante um período de tempo e área de abrangência, observando rastros deixados no percurso histórico de suas produções. Esses termos são comumente utilizados como sinônimos em variadas investigações, apesar das diferenças existentes. Na segunda parte é apresentado um balanço do que foi produzido entre 2003 e 2018, em quatro programas de pós-graduação de uma das universidades estaduais do Brasil, com tradição em investigação em Educação. Foram analisadas 54 produções (11 teses e 43 dissertações) referentes à Educação Infantil, desvelando seus achados e lacunas que poderão ser preenchidas em futuras pesquisas. O texto, busca demarcar as congruências e as divergências das concepções apresentadas e explicitar os percursos delineados no processo de construção do conhecimento na área.

Palavras-chave: estado da arte, estado do conhecimento, educação infantil.

Abstract: The article begins with a theoretical discussion of what are the critical reviews of the modes of production in the field of education recognized as "State of Art" and "State of Knowledge". They are studies that systematize what was produced during a period of time and area of coverage, observing traces left in the historical path of their productions. These terms are commonly used as synonyms in various investigations, despite the existing differences. In the second part is presented a balance of what was produced between 2003 and 2018 , in four graduate programs of one of the state universities in the country, with tradition in research in education. We analyzed 54 productions ( 11 theses and 43 dissertations) referring to Early Childhood Education, revealing their findings and gaps that may be filled in future research. The text seeks to demarcate the congruences and divergences of the conceptions presented and explain the paths outlined in the process of knowledge construction in the area.

Keywords: state of art, state of knowledge, early childhood education.

Resumen: El artículo inicia con una discusión teórica de cuáles son las revisiones críticas de los modos de producción en el campo de la educación reconocidos como "State of Art" y "State of Knowledge". Son estudios que sistematizan lo que se produjo durante un periodo de tiempo y área de cobertura, observando las huellas dejadas en la trayectoria histórica de sus producciones. Estos términos se utilizan comúnmente como sinónimos en várias investigaciones, a pesar de las diferencias existentes. En la segunda parte se presenta un balance de lo que se produjo entre 2003 y 2018 , en cuatro programas de postgrado de una de las universidades estatales del país, con tradición en investigación en educación. Se analizaron 54 producciones (11 tesis y 43 disertaciones) referidas a la Educación Infantil, revelando sus hallazgos y lagunas que pueden ser llenados en investigaciones futuras. El texto busca demarcar las congruencias y divergencias de las concepciones presentadas y explicar los caminos trazados en el proceso de construcción del conocimiento en el área.

Palabras clave: estado del arte, estado del conocimiento, educación infantil. 
Quando fazemos uma revisão bibliográfica em alguma pesquisa científica, sempre nos perguntamos: o que é isso mesmo que estamos fazendo? Este tipo de estudo serve para quê? Será que uma visão panorâmica da produção científica, sobre algum tema, favorece a maior compreensão e a avaliação da relevância desse tema e nos aponta lacunas, contradições, diversidades metodológicas, distintas modalidades de construção do conhecimento? Acreditamos que sim. Por essa razão, decidimos nos debruçar pelas diferentes modalidades reconhecidas para esse tipo de investigação. Encontramos, assim, duas denominações possiveis e, por isso, vamos analisá-las.

O Estado da Arte e o Estado do Conhecimento são denominações de levantamentos sistemáticos ou balanço sobre algum conhecimento, produzido durante um determinado periodo e área de abrangência. Dessa forma, os pesquisadores que decidem fazer um Estado da Arte ou Estado do Conhecimento têm em comum o objetivo de "olhar para trás", rever caminhos percorridos, portanto possiveis de serem mais uma vez visitados por novas pesquisas, de modo a favorecer a sistematização, a organização e o acesso às produções cientíicas e à democratização do conhecimento.

No Brasil, as terminologias "Estado da Arte" e "Estado do Conhecimento" têm sido utilizadas como sinônimo em diferentes e variadas pesquisas. Entretanto, isso não é consenso e por isso traremos alguns autores para validar tais posições.

Nas diversas universidades do País, no campo da Educação, diferentes pesquisas têm se propagado, acompanhando o mercado da produção científica e as demandas dos órgãos de financiamento. A grande maioria das investigações têm sido realizada no interior dos programas de pós-graduação, em dissertações de mestrado e teses de doutorado. Esses trabalhos encontram-se disponiveis nos arquivos das bibliotecas e, atualmente, nos respectivos programas digitais. $O$ acúmulo da produção de dissertações e teses, além da facilidade de acesso aos bancos de dados on-line inspiraram o desenvolvimento deste Dossiê.

Com o intuito de compreender como se dá o desenvolvimento das pesquisas e a construção do conhecimento, como ele se delineia e se configura na promoção de novas investigações; tais estudos são propostos para possibilitar a análise critica apontando tendências, congruências e divergências subjacentes. O acompanhamento permanente das produções científicas é fundamental e não deve ter uma terminalidade. Ao contrário, deve apresentar uma continuidade, traduzida pela tessitura paralela à sua construção, identificando e explicitando o percurso das pesquisas. Assim, o processo de construção do conhecimento é revelado para que se possa tentar a integração de resultados, identificar duplicações e lacunas na área, com o objetivo de estimular a produção de novas investigações.

\section{O Estado da Arte: a partir da visão de seus pesquisadores}

Segundo Brandão et al. (1986, p. 7), o termo "Estado da Arte" é originário da literatura científica americana e tem por meta "realizar levantamentos do que se conhece sobre um determinado assunto a partir de pesquisas realizadas em uma determinada área".

Para Ferreira (2002, p. 258), o "Estado da Arte" traz o desafio de ir além do mapeamento das produções cientíicas em diferentes campos do conhecimento, épocas e territórios, essa metodologia de caráter inventariante e descritiva busca conhecer "em que condições as teses, dissertações, publicações em periódicos, comunicações em anais de congressos e seminários têm sido produzidas".

Para Romanowsky e Ens (2006) o "Estado da Arte" que tinha por objetivo acompanhar a evolução do conhecimento científico durante certo período de tempo, acabou por se tornar um marco histórico. Esse termo tem sido utilizado nas pesquisas bibliográficas na área da Educação, Ciências Sociais e Saúde.

Assim sendo, o Estado da Arte resulta de um vasto acervo de diferentes tipos de pesquisas, com ênfases, graus de aprofundamento e registros diversos. Essa modalidade de revisão bibliográfica nos permite um diálogo com os demais pesquisadores de áreas afins e nos revela a riqueza de dados produzidas em suas 
pesquisas. Sposito (2009, p. 40) afirma que são raros os autores "que constroem problemáticas a partir de um diálogo horizontal com outros pesquisadores que escrevem sobre o tema, o que dificulta a acumulação também horizontal do processo de conhecimento".

Embora recente, o uso dessa metodologia vem sendo defendida por autores que a consideram importante para acompanhar as mudanças nas ciências, demarcando diferentes vertentes e facetas sobre as quais o conhecimento científico vem se constituindo. Traremos abaixo, algumas reflexões sobre o tema.

Romanowski (1999) na tese de doutorado "As Licenciaturas no Brasil: um balanço das teses e dissertações nos anos 90" realizou um Estado da Arte sobre teses e dissertações defendidas nos programas de pós-graduação em Educação no Brasil, no periodo de 1990-1998. Um de seus objetivos foi compreender como se deu a produção sobre a formação inicial de professores, em especial, nos cursos de licenciatura. Para a realização da pesquisa se consultou a base de dados no CD room da ANPED. Foram analisadas 39 teses e dissertações e 107 resumos. As pesquisas selecionadas foram obtidas nas bibliotecas dos programas de pós-graduação pelo sistema COMUT. ${ }^{3}$ Outro objetivo do estudo foi situar os cursos de licenciatura no quadro de expansão do ensino superior no Brasil, procurando perceber as problemáticas e as implicações dessa ampliação. A pesquisa concluiu que a melhoria na formação dos professores constitui-se em um desafio permanente, em especial no momento em que se apresenta uma nova proposta para os referidos cursos, exigindo a realização de pesquisas que avaliem tais mudanças.

No mesmo ano (1999), Brzenziski e colaboradores, na publicação "O Estado da Arte da Formação de Professores no Brasil", analisaram a temática nas dissertações e teses defendidas nos programas de pós-graduação em Educação do país, de 1990 a 1996, de artigos publicados em 10 periódicos da área, no periodo 1990-1997 e das pesquisas apresentadas no Grupo de Trabalho Formação de Professores da Associação Nacional de Pós-Graduação e Pesquisa em Educação (Anped), na década de 1990.

Os autores realizaram a Análise do Conteúdo de 115 artigos, de 284 dissertações e teses e de 70 trabalhos apresentados do GT (Grupo de Trabalho) Formação de Professores da ANPED, na década de 1990. Esse trabalho permitiu identificar preocupação com o preparo do professor para atuar nas séries iniciais do Ensino Fundamental. Evidenciou ainda o silêncio quase total em relação à formação do professor do Ensino Superior, Educação de Jovens e Adultos e do ensino técnico e rural, assim como a atuação de movimentos sociais e a literatura referente às crianças em situação de risco.

No trabalho de Sposito (2009) intitulado "Os jovens na Pós-Graduação: um balanço da produção discente em Educação, Serviço Social e Ciências Sociais (1999-2006)", a autora propõe um Estado da Arte do conhecimento produzido no campo da juventude daquele periodo. Analisou 1.427 teses e dissertações, a partir dos três campos mencionados no título do capitulo. Ajustificativa deu-se não só pela emergência do campo de estudos sobre juventude, mas na estruturação e na consolidação dele.

O estudo permitiu reconhecer os principais resultados das investigações, identificar as temáticas e as abordagens dominantes e fazer emergir temas necessários de serem investigados em pesquisas futuras. A autora destacou a presença de lacunas no acúmulo de conhecimentos sobre juventude que precisariam ser preenchidas por novos estudos. Outro problema registrado foi que os trabalhos não dialogavam entre si, pois ainda partiam de interesses isolados de pesquisadores dos diferentes programas de pós-graduação. Nessa direção, sugere que cabe aos orientadores ter uma atitude mais ativa, ajudando os alunos a reconhecer que a produção do conhecimento não se esgota em um único texto, ao contrário, precisa de interlocução e de continuidade a

COMUT é um Programa de Comutação Bibliográfica que permite o acesso às produções científicas disponibilizadas nas principais bibliotecas brasileiras. 
fim de que as questões de investigação sejam aprofundadas e ampliadas.

A autora (2009), na pesquisa O Estado da Arte sobre Juventude na Pós-Graduação Brasileira: Educação, Ciências Sociais e Serviço Social (19992006) defende a importância de estudos que adotem essa metodologia, pois ela não se limita a identificar as principais temáticas e enfoques presentes. Esse tipo de estudo é amplo e abrangente, pois leva em conta desde os resumos de artigos publicados em periódicos e eventos, até a produção de teses e dissertações. Isso possibilita estabelecer intercâmbio com outras áreas do conhecimento, analisando como determinado tema é diferentemente abordado nas áreas, suas contribuições, contradições, desafios propostos e lacunas que precisam ser preenchidas com novos estudos. Esse tipo de aprofundamento da análise permite estabelecer relações com diferentes produções bibliográficas (artigos, teses, dissertações e publicações em anais de eventos) em determinada área ou favorecer o diálogo entre diferentes campos do saber.

Autores como Sposito (2009), Brzenzinski (1999) e Romanowski (1999) utilizam o termo "Estado da Arte" nos levantamentos que realizam sobre a produção científica de seus campos de conhecimento. Outros autores preferem adotar a terminologia Estado do Conhecimento.

\section{O Estado do Conhecimento e alguns de seus pesquisadores}

Côco (2010), Ferreira (1999), Soares e Maciel (2000) Rocha (1999, 2001) adotam a terminologia "Estado do Conhecimento". Traremos alguns de seus trabalhos, assim identificados, para entender a razão da escolha e a preferência por essa terminologia.

Para estabelecer a diferença entre os termos utilizados nesse tipo de levantamento e análise, Soares e Maciel (2000) defendem que o "Estado do Conhecimento" é uma metodologia mais restrita, definindo-a como um estudo que aborda apenas um setor das publicações sobre um determinado tema. Segundo as autoras, a multiplicidade de trabalhos nas diferentes áreas e nas ênfases diversas, não colaboram para in- tegrar as pesquisas e seus resultados, além de não explicar as contradições e as incoerências encontradas. Antes disso, um primeiro passo a ser dado é elaborar um "Estado do Conhecimento" na área selecionada, uma revisão crítica da literatura especifica, com a identificação dos aspectos que têm sido valorizados e os referenciais teóricos que vêm subsidiando as pesquisas nos últimos anos. No caso do estudo intitulado "O Estado do Conhecimento em Alfabetização 1961-1989" foram analisadas teses e dissertações nas áreas de Educação, Psicologia, Letras e Distúrbios da Comunicação. Soares e Maciel (2000), no livro Alfabetização - Série Estado do Conhecimento, registraram as produções acadêmico-científicas sobre a aquisição da língua escrita por crianças, no processo de escolarização regular. A descrição se deu à luz de determinadas categorias: os temas privilegiados, os referenciais teóricos adotados; além dos gêneros em que o conhecimento produzido se expressava. Procuraram, ainda, indicar as relações presentes nessas categorias e evidenciar, em uma perspectiva histórica, a existência maior ou menor, ao longo do periodo analisado, dos diferentes temas, referenciais teóricos e gêneros.

As autoras tinham por objetivo apresentar estudos e oferecer subsídios para a definição de uma política voltada ao incentivo à pesquisa nessa área. Detectaram também lacunas, apontaram temas ausentes ou insuficientemente explorados; além de referenciais teóricos cuja produção sobre alfabetização era pouco significativa e, também, apresentar alternativas metodológicas de investigação que, embora promissoras, ainda eram pouco utilizadas em pesquisas sobre o tema.

De acordo com Romanowski e Ens (2006), estudos sobre o Estado do Conhecimento:

\footnotetext{
(...) podem significar uma contribuição importante na constituição do campo teórico de uma área de conhecimento, pois procuram identificar os aportes significativos da construção da teoria e prática pedagógica, apontar as restrições sobre o campo em que se move a pesquisa, as suas lacunas de disseminação. identificar experiências inovadoras investigadas que apontem alternativas de solução para os problemas da prática e reconhecer as contribuições da pesquisa na constituição de propostas na área focalizada. (p. 39)
} 
Para os autores, a possibilidade de aprofundamento da análise é fundamental "para a definição de um campo investigativo em tempos de intensas mudanças associadas aos avanços crescentes da ciência e da tecnologia" (Romanowski \& Ens, 2006, p. 39).

A defesa da metodologia Estado do Conhecimento é uma tentativa de melhor ajustar os objetivos às especificidades dos variados campos de investigação, corroborando intencionalmente para a contextualização, a problematização e a exploração de desafios e orientação de abordagens futuras (Torres \& Palhares, 2014). Autores como Vosgerau e Romanowski (2014) afirmam que as revisões na literatura científica podem contribuir para conhecer o percurso histórico das pesquisas de uma área ou um tema a ser consolidado.

Côco (2010), na pesquisa "Mapeamento da Educação Infantil no Espírito Santo" buscou desvendar o cenário da Educação Infantil naquele estado. O levantamento se deu a partir do material produzido no Programa de Pós-Graduação em Educação da Universidade Federal do Espirito Santo (PPGE/UFES), tomando como referência a totalidade das produções do Programa e, nela, a evolução da produção relativa ao tema - Educação Infantil (teses e dissertações).

A pesquisa apontou múltiplas conquistas e desafios a serem enfrentados no atendimento às crianças pequenas que vão desde a luta pelo pertencimento da Educação Infantil à dinâmica educacional, as articulações de ações integradas com outras secretarias municipais, o aumento de ofertas de vagas, até a necessidade de qualificar o trabalho dos profissionais da área, integrando as ações de cuidado e de educação que favoreçam o desenvolvimento de uma concepção de Educação Infantil como espaço privilegiado de aprendizagem, além de ampliar as experiências de todos os sujeitos envolvidos.

Ferreira (2001) na A pesquisa sobre leitura no Brasil: 1980-1995 leu e interrogou os resumos das pesquisas desenvolvidas no periodo estudado, nas faculdades de Letras/Linguistica, Biblioteconomia, Comunicações, Educação e Psicologia. A primeira parte do trabalho objetivou inventariar, analisare descrever a trajetória de leitura, enquanto campo de produção de conhecimentos no Brasil, destacando: anos de publicação, locais de produção, áreas em que se originaram as pesquisas; gênero/ sexo dos pesquisadores e focos temáticos. $\mathrm{Na}$ segunda parte, a autora tomou os resumos como gêneros de discurso acadêmico e os examinou como objeto de aprofundamento do estudo.

O estudo de Rocha et al. (2001) intitulado "Série Estado do Conhecimento n ${ }^{\circ} 2$ - Educação Infantil 1983-1996, ampliou a pesquisa anterior realizada em 1999 e buscou analisar a produção cientifica sobre a Educação Infantil no Brasil, a partir dos programas de pós-graduação em Educação, no período citado. O estudo teve por objetivo consolidar e integrar um conjunto de dados sobre esse campo específico da Educação, o que resultou na elaboração de um banco de dados com 432 registros cuja intenção foi permitir a atualização constante de informações e a sua ampla divulgação. O trabalho apresentou uma visão geral da produção do conhecimento sobre a educação de crianças de 0 a 6 anos, tendo em vista aspectos gerais da trajetória da área, no periodo de 14 anos.

Ferreira (2002), no artigo "As pesquisas denominadas: O Estado do Conhecimento" enfatiza que o que mobiliza os pesquisadores nesse tipo de inventário descritivo da produção acadêmica é o não conhecimento da totalidade de produções em determinada área que apresenta crescimento quantitativo e qualitativo, principalmente, em relação às considerações tecidas nos programas de pós-graduação e, mesmo assim, ainda não foram amplamente divulgadas.

Os diversos autores citados apresentam orientações metodológicas distintas que podem vir a corroborar no reconhecimento e na identificação dos principais interesses dos estudos que serão aqui analisados. Sejam os que se reconhecem como autores de investigações do tipo Estado da Arte ou Estado do Conhecimento, todos ajudam a identificar as mais recentes publicações de temas variados referentes à Educação da infância brasileira, através da divulgação dos resultados e a emersão das possiveis lacunas existentes que poderão ser preenchidas com estudos futuros. 
Outra forma de identificar e compreender essa modalidade de pesquisa científica é nomeá-la de meta-investigação. Conforme salientam Torres e Palhares (2014), a meta-investigação é um empreendimento necessário

(...) na identificação de padrões de pesquisa, no levantamento de tendências dominantes e na exploração de desafios e orientações para abordagens futuras. Se, por um lado a literatura abre caminho ao conhecimento dos itinerários, por outro, pouco sabemos sobre a forma como, na realidade concreta, se constroem os objetos de estudo, se formulam os problemas, se escolhem os métodos e se aplicam as técnicas. (p. 15)

O que aproxima esses estudiosos é o fato de buscarem o que já foi produzido, desvelar seus principais interesses, seguir os percursos metodológicos adotados, expor os hiatos existentes e, ainda, revelar o volume de trabalhos produzidos, valorizando-os, fazendo-os emergir ao serem propagados (Ferreira, 2002).

A próxima seção apresenta o levantamento dos estudos científicos produzidos nos programas de pós-graduação em Educação da Universidade do Estado do Rio de Janeiro (UERJ) nos últimos anos, que tiveram como eixo central de investigação a Educação Infantil.

\section{O lugar da Educação Infantil nas produções científicas da UERJ}

Iniciamos este artigo com reflexões propostas por Pimentel (2016), na pesquisa realizada do tipo Estado do Conhecimento referente aos estudos sobre Educação Infantil produzidos no universo das dissertações e das teses de quatro programas de mestrado e doutorado de Educação, da Universidade do Estado do Rio de Janeiro (UERJ). São eles: Processos Formativos e Desigualdades Sociais (PPGEDU); Educação, Cultura e Comunicação em Periferias Urbanas (PPGECC); Políticas Públicas e Formação Humana (PPFH) e Programa de Pós-Graduação em Educação (Proped).

A autora identificou os programas de pós-graduação, em geral, como espaços institucionais de pesquisa que promovem e fortalecem a construção do conhecimento, a partir de perspectivas distintas.
No interior deles há linhas e grupos de pesquisa que se constituem em espaços de reflexão, discussão e produção de conhecimento; reunindo pesquisadores, abordagens teórico-metodológicas e referenciais teóricos diferenciados. Todos contribuem para a constituição, ampliação e demarcação de distintos olhares para o campo da Educação.

Naquela pesquisa, Pimentel (2016) privilegiou as produções acadêmicas referentes à Educação Infantil. Buscou identificar as temáticas relevantes, as metodologias adotadas e os referenciais teóricos mais frequentes nos distintos programas. O interesse pelo campo dos Estudos da Infância mostrou-se presente, por ser uma área que tem se expandido nas últimas décadas. Diferentes temáticas têm sido exploradas, acompanhando o esforço de pesquisadores da Educação Infantil em promover e mobilizar o debate, a reflexão e a discussão sobre o tema. Tudo isso contribui para a ampliação e a consolidação do campo de estudo.

Para a realização da pesquisa a autora elaborou algumas questões iniciais que orientaram a trajetória: quais as produções científicas elaboradas no periodo de 2003-2015 que têm a criança como foco? Qual o lugar ocupado pela criança de 0-5 anos? Que temáticas são mais exploradas? Que outros temas precisam ser intensificados? As indagações levaram a pesquisadora a trilhar caminhos especificos de uma pesquisa bibliográfica que adotasse alguns procedimentos que servissem de bússola para alcançar os objetivos da investigação.

O primeiro passo foi buscar teses e dissertações, a partir do termo mais amplo "Infância", na base de dados da biblioteca virtual da UERJ, localizada no portal da Coordenação de Aperfeiçoamento de Pessoal de Nivel Superior (Capes). O segundo, foi centrar no termo Educação Infantil e finalizar com trabalhos específicos sobre creche. Para este artigo, a segunda autora, ampliou a análise, incluindo as produções dos referidos programas, nos anos de 2016 a 2018.

Os dados que serão aqui analisados referem-se a soma das duas buscas. No âmbito dos sites dos programas pesquisados foram encontradas 104 (cento e quatro) produções; sendo 23 teses e 81 dissertações. Privilegiaremos aqui as 54 
produções (11 teses e 43 dissertações) referentes à Educação Infantil.

Os artigos trilham um terreno de constantes reafirmações de direitos e discussões sobre o lugar da Educação Infantil e sua consolidação como campo de estudos fértil para o desenvolvimento de investigações. O trabalho de análise adotou o seguinte procedimento: i) leitura do título, palavras-chave e resumo; ii) leitura minuciosa do resumo e, posterior, sintese do mesmo o que possibilitou a familiarização com cada temática e a apropriação do seu conteúdo. A produção científica referente à "Educação Infantil" foi dividida nos seguintes eixos temáticos: Formação de Profissionais, Políticas Públicas, Estudos Biográficos, Curriculo, Diversidade (relações étnico-raciais, inclusão, sexualidade), Legitimação da Infância como Experiência e Revisão Bibliográfica.

Os 12 (doze) estudos sobre Formação de Profissionais de Educação Infantil (Frangella, 2006; Fabricante, 2012; Fernandes, 2012; Matos, 2013; Carpi, 2014: Campos, 2014; Zadminas, 2016; Abreu, 2017; Oliveira, 2017; Morais, 2018; Silva, 2018; Santos, 2018) trazem preocupações, reflexões e perspectivas que perpassam, desde o currículo oferecido na formação dos profissionais da infância até a construção de práticas no cotidiano das creches e pré-escolas. Os trabalhos reunidos apontam a formação dos profissionais como aspecto imprescindivel à caracterização do trabalho docente na Educação Infantil.

O Currículo desenvolvido na Educação infantil é questão intrinseca e complementar às discussões sobre a formação dos profissionais da área. O momento histórico vivido repleto de polêmicas, instiga e enfrenta as discussões sobre o currículo na Educação Infantil. A análise sobre o tema se deu a partir de 12 (doze) estudos (Cardoso, 2003; Maia, 2011; Drumond, 2014; Silva, J., 2016; Silva, M., 2016; Silva, O., 2016; Pires, 2016; Alves, 2016; Lima, 2016; Almeida, 2017; Santos, 2017; Dinis, 2018) que ofereciam subsidios para pensar a construção de currículos de Educação Infantil.

No viés das Políticas Públicas para a criança pequena, 14 (quatorze) estudos (Ruani, 2007; D’almeida 2009; 2014; Brasil, 2016; Lamare, 2016,
Coutinho, 2017; Endlich, 2017; Oliveira, 2017; Peres, 2017; Faria, 2018; Gil, 2018; Mendes, 2018; Ribeiro, 2018; Rodrigues, 2018) analisam o quanto os direitos das crianças à Educação Infantil são atravessados por interesses políticos e explicitam o lugar que as infâncias e a educação destinadas a elas ocupam em nossos dias.

No eixo Estudos Biográficos, Carvalho (2014) e Reis (2014) ajudam a dar visibilidade e contribuem para reafirmar a importância de educadoras que deixaram o seu legado para a Educação Infantil no Brasil ao longo da história. Já Elia (2018) analisou como Mário de Andrade pôs em prática um projeto de Educação Infantil, denominado "Parques Infantis", com crianças realizando atividades artísticas ao ar livre, inspiradas em diversas manifestações culturais do Brasil.

A produção acadêmica sobre as questões da Diversidade, na área da Educação Infantil, cresceu nos programas de pós-graduação em Educação/ UERJ. Entretanto, no levantamento realizado, apenas 8 (oito) trabalhos foram encontrados, o que caracteriza ainda haver uma baixa produção sobre o tema (Pereira, 2012; Campos, 2012; Rosa, 2012; Braga, 2016; Siqueira, 2017; Rossato, 2017; Miceli, 2017; Santos, 2018). Eles representam três campos de análise: relações étnico-racionais, inclusão e sexualidade. A seguir, a descrição do trabalho em ordem:

Pereira (2012), analisou os significados expressos nas enunciações de professoras e de estudantes de Pedagogia sobre as relações étnico-raciais. Braga (2016) investigou as primeiras experiências das crianças negras com seus cabelos crespos na Educação Infantil. Na mesma linha Miceli (2017) realizou um estudo das relações étnico-raciais e teve como campo uma Educação Infantil universitária. De forma semelhante Santos (2018) analisou como as relações étnico-raciais apareciam representadas nos espaços de uma unidade de Educação Infantil em Juiz de Fora. As quatro pesquisas ressaltaram o importante papel do professor para uma educação antirracista.

A tese de Campos (2012) acompanhou o processo de inclusão de uma criança com Síndrome de Down em uma turma regular de Educação Infantil e Siqueira (2017) verificou os efeitos da for- 
mação continuada de ação colaborativa com uma professora de Sala de Recursos Multifuncionais, para o uso da Comunicação Alternativa e Ampliada nas trocas comunicativas e na interação de duas crianças com Transtorno do Espectro Autista, na Educação Infantil. A temática da sexualidade é tomada em duas investigações a saber: Rosa (2012) analisou como os homens se enunciam e são enunciados nos trabalhos da Educação Infantil e nas séries iniciais do ensino fundamental. A pesquisa de Rossato (2017) problematizou as aprendizagens de gênero-sexualidade a partir de atividades e experiências que emergiram de uma turma de Educação Infantil.

A legitimação da infância como experiência foi identificada em duas dissertações (Melro, 2013; Lopes, 2015). Melro (2013) buscou compreender como a criança e a infância eram consideradas no campo acadêmico tradicional e como são vistas, compreendidas e legitimadas na contemporaneidade. A pesquisa apresentou caminhos possiveis para pensar em uma escola de Educação Infantil, em que professores e crianças compartilham, intencionalmente, a experiência educativa. Já o estudo de Lopes (2015) apresenta a infância muito além do tempo cronológico, levando os sujeitos a estarem abertos ao novo e para o mundo. A escola é feita das experiências das crianças, rompendo com formas padrão de ensino, levando adultos e crianças a aprenderem a ler e a decifrar o mundo de maneira individual e coletiva.

A Revisão Bibliográfica foi objeto de estudo de 3 (três) trabalhos, sendo 1 (uma) tese e 2 (duas) dissertações. Miranda (2016) analisou a produção acadêmica sobre Educação Infantil em contextos rurais no período de 2000 a 2010. O trabalho de Dornelles (2016) investigou a relação entre criança, livro e literatura infantil a partir de ações acadêmicas desenvolvidas em uma Biblioteca Escolar, localizada nas dependências de uma creche universitária. A pesquisa de Pimentel (2016) ${ }^{4}$ analisou teses e dissertações de quatro programas de pós-graduação em Educação da UERJ, no período de 2003 a 2015, a partir dos temas: Infância,
Educação Infantil e Creche. Na dissertação o tema creche foi aprofundado e analisado criticamente. A aproximação com as familias e a formação dos profissionais que atuam com bebês e crianças pequenas assumiram centralidade.

Ao buscar sintetizar as pesquisas realizadas nos quatro programas de pós-graduação em Educação da UERJ, ao longo de duas décadas (2003-2018), busca-se demarcar as congruências e as divergências das concepções apresentadas e explicitar os percursos delineados no processo de construção do conhecimento. Nessa direção, um estudo bibliográfico como este aqui representado como "Estado do Conhecimento" demarca historicamente o lugar da Universidade e seus pesquisadores, não só no compromisso com a formação inicial de professores da infância do nosso Pais, mas também na produção do conhecimento, através de pesquisas que potenciam a Educação Infantil como direito das crianças pequenas e ajudam na construção de práticas de qualidade para esse segmento.

As análises apontam que algumas discussões merecem aprofundamento por ainda estarem silenciadas nas pesquisas da Educação Infantil. São temáticas que alertam para as relações étnico-raciais, inclusão de crianças com deficiência, sexualidade e gênero. Nos estudos analisados apenas dois deixam clara a inclusão da participação direta das crianças e denunciam que quanto menor a criança, menores são as chances de suas vozes serem difundidas e ecoadas e que suas infâncias sejam reconhecidas, inclusive nas pesquisas.

As pesquisas analisadas por Pimentel (2016) e complementadas por Souza (2019) estavam interessadas em revelar os desafios a serem percorridos na consolidação de uma pedagogia especifica para a Educação Infantil (Vasconcellos, 2008). O compromisso foi dar tratamento especial às produções recentes, referendando os autores que se debruçam a pesquisar a primeira infância, fazendo emergir seus principais interesses, arcabouço teórico e concepções centrais em que se baseiam. Os resultados apontam que os artigos apresentados

4 Parcialmente aqui apresentada. 
se constituem em trabalhos complexos sobre corpus de análise que exigem tratamentos refinados, olhares críticos e aprofundados.

Para além dessas especificidades, as revisões bibliográficas do tipo "Estado do Conhecimento, Estado da Arte ou Meta-Investigação" são importantes instrumentos que contribuem no acompanhamento histórico da produção do conhecimento, demarcando temas ainda pouco estudados e favorecem o intercâmbio entre os diferentes campos do saber.

\section{Referências}

Abreu, R. S. (2017). Tia, eu fiz um desenho prá você! Investigando o desenho infantil a partir das narrativas de crianças pequenas e da prática pedagógica (Dissertação de mestrado). Universidade do Estado do Rio de Janeiro, São Gonçalo, Brasil.

Almeida, A. G. de. (2017). A Roda de Conversa na escola da infância: (re) pensando as experiências de linguagens no cotidiano de uma unidade municipal de Educação Infantil (Dissertação de mestrado). Universidade do Estado do Rio de Janeiro, São Gonçalo, Brasil.

Alves, F. de S. (2016). "Tem uma hora que aprendemos a contar na cabeça": um estudo sobre a construção do número e o campo aditivo na educação infantil (Dissertação de mestrado). Universidade do Estado do Rio de Janeiro, Duque de Caxias, Brasil.

Aquino, L; M; L. Vasconcellos, V.; M.; R. (2011). Orientação Curricular para a Educação Infantil: Referencial Curricular Nacional e Diretrizes Curriculares Nacionais. In Vasconcellos, V. M. R. (Org.), Educação da Infância: História e Politica (2. ed.). Niterói: Editora da UFF.

Braga, A. de O. (2016). "Solta o cabelo!": etnografia sobre o cabelo crespo como marcador de identidade étnico-racial entre crianças negras da Educação Infantil (Dissertação de Mestrado). Universidade do Estado do Rio de Janeiro, Duque de Caxias, Brasil.

Brandão, Z:; Baeta, A. M. B; Rocha, A. D. C. (1986). Evasão e repetência no Brasil: a escola em questão (2. ed.). Rio de Janeiro, RJ: Dois Pontos.

Brasil, M. G. de P. (2016). Espaço(s) na educação infantil: entre politicas e práticas (Tese de doutorado). Faculdade de Educação, Universidade do Estado do Rio de Janeiro, Rio de Janeiro, Brasil.

Brzezinski, Iria. (2013). Estado do Conhecimento Sobre Formação de Profissionais da Educação: teses e dissertações do período 2003-2010. Indagatio Didactica, 5(2).

Carvalho, L. D. (2014) Caminhos de vida, percursos na educação da Infância: um estudo sobre a trajetória profissional da educadora Astrogildes Delgado de Carvalho (anos 1940-1980) (Tese de doutorado). Pós-graduação em Educação, Universidade do Estado do Rio de Janeiro, Rio de Janeiro, Brasil.
Carpi, C. M. S. (2014). Tornar-se Docente: uma viagem pelas experiências formativas de professores da Educação Infantil do Centro Educacional de Niterói de 1980 a 2006 (Dissertação de mestrado). Pós-graduação em Educação, Universidade do Estado do Rio de Janeiro, São Gonçalo.

Campos. K. P. B. (2012). Isabel na escola: desafios e prospectivas para a inclusão de uma criança com sindrome de down numa classe comum (Tese de doutorado). Pós-Graduação em Educação, Universidade do Estado do Rio de Janeiro, Rio de Janeiro, Brasil.

Cardoso, M. A. (2003). Cresça e Apareça: um estudo de caso sobre a construção da autonomia na Educação Infantil (Dissertação de mestrado). Pós-Graduação em Educação, Universidade do Estado do Rio de Janeiro, Rio de Janeiro.

Campos, M. I. F. (2014). O PROINFANTIL no municipio do Rio de Janeiro: concepções de criança nos Projetos de Estudos (Dissertação de mestrado). Pós-Graduação em Educação, Universidade do Estado do Rio de Janeiro, Rio de Janeiro, Brasil.

Chervel, A. (1990). História das Disciplinas Escolares: reflexões sobre um campo de pesquisa. Teoria \& Educação, 2, 177-229.

Côco, V. A. (2010) Configuração do trabalho docente na Educação Infantil. Espirito Santo. Recuperado de http://www.anpae.org.br/iberolusobrasileiro2010/ cdrom/118.pdf

Côco, V. (2010). A configuração do trabalho docente na educação infantil. In Congresso Ibero-Luso Brasileiro de Política e Administração da Educação.

Costa. T. G. (2011). Movimentos Sociais e direito à educação infantil em São Gonçalo: perspectivas, dilemas da politica dos convênios do poder público e creches comunitárias (Dissertação de mestrado). Pós-Graduação em Educação, Universidade do Estado do Rio de Janeiro, São Gonçalo, Brasil.

Coutinho, A. L. (2017). Politicas de curriculo: relação familia e escola nos textos políticos para Educação Infantil (Dissertação de mestrado). Faculdade de Educação, Universidade do Estado do Rio de Janeiro, Rio de Janeiro, Brasil.

D'almeida, K. P. de M. (2009). Educação Infantil e Direito: práticas de controle como campo de análise (Dissertação de mestrado). Universidade do Estado do Rio de Janeiro, Brasil.

D'almeida, K. P. de M. (2014). A obrigatoriedade da educação infantil: governamentalidade e refinamento das técnicas de governo (Tese de doutorado). Pós-Graduação em Educação, Universidade do Estado do Rio de Janeiro, Brasil.

Dinis, A. C. (2018). Representação semiótica: uma perspectiva para a construção do conceito de número na educação infantil (Dissertação de mestrado) Universidade do Estado do Rio de Janeiro, Duque de Caxias, Brasil.

Dornelles, P. de O. (2016) A Creche UFF e sua Flor de Papel - uma análise sobre a produção de conhecimento de uma biblioteca escolar infantil (Dissertação de mestrado. Faculdade de Educação, Universidade do Estado do Rio de Janeiro, Rio de Janeiro, Brasil. 
Drumond, R. C. R. (2014). Educação Infantil- Ensino Fundamental: possibilidades de produções curriculares no entre-lugar (Dissertação de mestrado). Pós-Graduação em Educação, Universidade do Estado do Rio de Janeiro, Duque de Caxias, Brasil.

Elia, R. (2018). Um turista aprendiz nos Parques Infantis: Mário de Andrade, viagem e educação (Dissertação de mestrado). Faculdade de Educação, Universidade do Estado do Rio de Janeiro, Rio de Janeiro, Brasil.

Endlich, A. R. F. G. (2017). Ambientes para a Educação Infantil: o Proinfância em Quatis (Dissertação de mestrado). Faculdade de Educação, Universidade do Estado do Rio de Janeiro, Rio de Janeiro, Brasil.

Fabricante, B. S. (2012) Investigando caminhos formativos de professoras da(s) infância(s) em Rio Bonito: o curso normal em questão (Dissertação de mestrado). Pós-Graduação em Educação, Universidade do estado do Rio de Janeiro, São Gonçalo, Brasil.

Faria, C. E. C. (2018). Políticas Públicas de Educação Infantil no municipio de Duque de Caxias - RJ: de 2007 a 2017 (Dissertação de mestrado). Faculdade de Educação, Universidade do Estado do Rio de Janeiro, Rio de Janeiro, Brasil.

Fernandes, L. S. (2012). O portfólio na formação docente como espaço de produção curricular na Educação Infantil: o PROINFANTIL em Mesquita - RJ (Dissertação de mestrado). Pós-Graduação em Educação, Universidade do Estado do Rio de Janeiro, Duque de Caxias, Brasil.

Ferreira, N. S. de Almeida. As pesquisas denominadas "estado da arte". Educação \& Sociedade, 79, 2002.

Ferreira, N. S. de Almeida et al. (2001). A pesquisa sobre leitura no Brasil: 1980-1995 (1. ed.). Campinas: Komedi: Arte e Escrita

Frangella. R. C. P. (2006). Na procura de um curso: currículo-formação de professores-Educação Infantil Identidade(s) em (des)construção (?) (Tese de doutorado). Pós-Graduação em Educação, Universidade do Estado do Rio de Janeiro, Rio de Janeiro. Brasil.

Gatti, B. A. (2002). A construção da Pesquisa em Educação no Brasil. Brasília: Plano Editora.

Gil, M. de O. G. (2018). Políticas Públicas de Educação Infantil no Municipio do Rio de Janeiro: Berçário em Foco (2009 - 2016) (Tese de doutorado). Faculdade de Educação, Universidade do Estado do Rio de Janeiro, Rio de Janeiro, Brasil.

Lamare, F. de F. de. (2016). Contradições na concepção de formação humana nas politicas de Educação Infantil no Brasil: o que revelam os documentos do periodo de 2003 a 2010 (Tese de doutorado). Programa de Políticas Públicas e Formação Humana, Universidade do Estado do Rio de Janeiro, Rio de Janeiro, Brasil.

Lima, F. F. de S. (2016). "9/5": Tempo(s) e Espaço(s) na Educação da Pequena Infância numa Escola Pública do Município de Niterói (Dissertação de mestrado). Faculdade de Formação de Professores. Programa de Pós-Graduação em Educação. Universidade do Estado do Rio de Janeiro. São Gonçalo, Brasil
Lopes, A. B. P. (2015). Habitar o presente, fazer um mundo: movimentos de crianças e adultos em uma escola de educação infantil. (Dissertação de mestrado). Pós-Graduação em Educação, Universidade do Estado do Rio de Janeiro, Brasil.

Maia, S. N. L. (2011). Leituras e produções de imagens no cotidiano de uma escola de Educação Infantil: possibilidades de construção de conhecimento (Dissertação de mestrado). Pós-Graduação em Educação, Universidade do Estado do Rio de Janeiro, São Gonçalo, Brasil.

Mattos, P. G. G. (2013). O curso de Pedagogia da Faculdade de Formação de Professores da UERJ a partir de narrativas de formação de professoras das infâncias (Dissertação de mestrado). Pós-Graduação em Educação, Universidade do Estado do Rio de Janeiro, São Gonçalo, Brasil.

Melro. R. dos S. (2013). Percursos de uma Educação de crianças: o que as vozes infantis têm a nos ensinar? (Dissertação de mestrado). Faculdade de Formação de Professores de São Gonçalo, Universidade do Estado do Rio de Janeiro, São Gonçalo.

Mendes, J. da S. (2018). Programa "Mais Infância": o direito ao atendimento educacional na primeira infância no munícipio de Niterói (Dissertação de mestrado). Faculdade de Formação de Professores, Universidade do Estado do Rio de Janeiro, São Gonçalo, Brasil.

Menezes, L.B. D de. (2010). Especialmente recomendado para menores de seis anos. (Dissertação de mestrado). Pós-Graduação em Educação, Universidade do Estado do Rio de Janeiro, Rio de Janeiro, Brasil.

Miceli, P. de A. M. (2017). Negritude nas Práticas Pedagógicas da EEI-UFRJ. Estudo das Relações Etnico-Raciais na Escola de Educação Infantil da UFRJ (Dissertação de mestrado). Faculdade em Educação, Universidade do Estado do Rio de Janeiro, Rio de Janeiro, Brasil.

Miranda, D. R. (2016). Educação Infantil em contextos rurais: perspectivas presentes na produção acadêmica da última década (2000-2010) (Tese de Doutorado). Centro de Educação e Humanidades, Universidade do Estado do Rio de Janeiro, Rio de Janeiro, Brasil.

Moraes, R. M. (2007). Conselhos Tutelares e Educação Infantil: Impasses Desafios e Tensões. Avanços e Retrocessos de uma Relação em Construção (Dissertação de mestrado). Pós-Graduação em Educação, Universidade do Estado do Rio de Janeiro, Brasil.

Morais, A. P. S. (2018) Por uma espreita infantil: fazer-se professora entre os movimentos do imperceptivel. (Dissertação de mestrado). Faculdade de Educação, Universidade do Estado do Rio de Janeiro, Rio de Janeiro, Brasil.

OLIVEIRA, R. T. (2017). Audiovisualidades praticadas nos/com os cotidianos da Educação Infantil como dispositivos para autoformação e formação docente continuada (Dissertação de mestrado). Centro de Educação e Humanidades, Universidade do Estado do Rio de Janeiro, Rio de Janeiro, Brasil.

OLIVEIRA, C. G. de. (2017). "Que rei sou eu?" Escolas Públicas de Excelência, Políticas Educacionais e Curriculo: uma análise sobre o processo de instituição da Educação Infantil no Colégio Pedro II (Tese de doutorado). Centro de Educação e Humanidades, Universidade do Estado do Rio de Janeiro, Rio de Janeiro, Brasil. 
Pereira. R. R. (2012). Pesquisa com Crianças. Infância em Pesquisa, v. 1.

Peres, S. P. V. (2017). Por Uma Politica Nacional Para a Educação Infantil: Os Cadernos da COEDI (1994 -1998) (Dissertação de mestrado). Universidade do Estado do Rio de Janeiro, São Gonçalo, Brasil

Pimentel, A. P. (2016). 12 anos (2003-2015) de Educação Infantil na UERJ: entre teses e dissertações (Dissertação de mestrado). Universidade do Estado do Rio de Janeiro, Rio de Janeiro, Brasil.

Pires, M. I. (2016). Educação Sexual: significações que circulam e disputam hegemonia em textos curriculares de Educação Infantil no Município de Duque de Caxias $R J$ (Dissertação de mestrado). Universidade do Estado do Rio de Janeiro, Duque de Caxias, Brasil.

Reis, A. C. C. (2014) Formação docente para a infância: o legado de Heloisa Marinho (1934 - 1938) (Dissertação de mestrado). Pós-Graduação em Educação, Universidade do Estado do Rio de Janeiro. Rio de Janeiro. Brasil.

Ribeiro, R. (2018) Uma análise do Programa "Mais Infância": concepções e ações envolvidas na implementação da politica pública para a Educação Infantil em Niterói (2013-2016) (Dissertação de mestrado). Faculdade de Formação de Professores, Universidade do Estado do Rio de Janeiro, São Gonçalo, Brasil.

Rocha, E. C. 1999. A Pesquisa em educação infantil no Brasil: trajetória recente e perspectivas de consolidação de uma pedagogia (Tese de doutorado). Universidade Estadual de Campinas, Campinas, Brasil.

Rocha, E. C; Silva Filho, J. J.; Strenzel, G. R. (Orgs.). (2001). Educação Infantil (1983-1996). Brasilia: MEC/ Inep/Comped. (Série Estado do Conhecimento n. 2). Recuperado de http://Www.inep.gov.br/download/ cibec/2001/estado_do_conhecimento/serie_doc_. educacão_infantil.pdf/

Rocha, E. C; Romanovski, J. P. (set./dez. 2006) As pesquisas denominadas do tipo o Estado da Arte em educação. Revista Diálogo Educacional, Curitiba, 6(19), 37-50.

Rodrigues, D. A. dos S. (2018). Mais Infância para quem? Problematizando o "Programa Mais Infância" a partir de vozes de crianças no cotidiano de uma Unidade Municipal de Educação Infantil em Niterói (Dissertação de mestrado). Faculdade de Formação de Professores, Universidade do Estado do Rio de Janeiro. São Gonçalo, Brasil.

Romanowski, J. P. (2002). As licenciaturas no Brasil: um balanço das teses e dissertações dos anos 9o. São Paulo: Faculdade de Educação da USP.

Romanowski, J. P \& ENS, Romilda Teodora. (2006). As pesquisas denominadas do tipo" Estado da Arte. Revista Diálogo Educacional, 6(19).

Rosa. F. J. P. da. (2012). O dispositivo da sexualidade enquanto enunciador do professor-homem no magistério das séries iniciais e na educação infantil (Dissertação de mestrado). Pós-Graduação em Educação, Universidade do Estado do Rio de Janeiro, Duque de Caxias, Brasil.
Rossato, B. C. L. (2017). Aprendizagens de gênero-sexualidade na/com a Educação Infantil: apontamentos para pensar os curriculos (Dissertação de mestrado). Faculdade de Educação, Universidade do Estado do Rio de Janeiro, Rio de Janeiro, 2017.

Santos, E. C. R. dos. (2018). Dança na Escola de Educação Infantil da UFRJ: proposta pedagógica e práticas docentes (Dissertação de mestrado). Faculdade de Educação, Universidade do Estado do Rio de Janeiro, Rio de Janeiro, Brasil.

Santos, R. (2018). Bidocência na Educação Infantil do Colégio Pedro Il: possibilidades e desafios narrados pelas professoras (Dissertação de mestrado). Faculdade de Formação de Professores, Universidade do Estado do Rio de Janeiro, São Gonçalo, Brasil.

Santos, A. (2018). A Educação das Relações Étnico-Raciais na Creche: uma leitura a partir do espaço institucional (Tese de doutorado). Universidade do Estado do Rio de Janeiro, Brasil.

Silva, J. V. da. (2016). Indagações sobre a relação entre curriculo e o uso das midias digitais: O projeto KidSmart na Educação Infantil do Municipio do Rio de Janeiro (Dissertação de mestrado). Pós-Graduação em Educação, Universidade do Estado do Rio de Janeiro. Rio de Janeiro, Brasil.

Silva, M. M. (2016). Formação do leitor literário na Educação Infantil (Tese de doutorado). Faculdade de Educação, Universidade do Estado do Rio de Janeiro, Rio de Janeiro, Brasil.

Silva, M. do N. (2016). O que aprendemos com os bebês? Uma experiência de pesquisa no berçário de uma creche pública de Niterói (Dissertação de mestrado). Faculdade de Formação de Professores, Programa de Pós-Graduação em Educação, Universidade do Estado do Rio de Janeiro, São Gonçalo, Brasil.

Silva, T. N. da. (2018). Escrever, refletir, compartilhar: marcas da formação continuada em registros de uma professora das infâncias (Dissertação de mestrado). Faculdade de Formação de Professores, Universidade do Estado do Rio de Janeiro, São Gonçalo, Brasil.

Siqueira, M. C. D. (2017) Todos na roda: o uso da Comunicação Alternativa e Ampliada em uma escola de Educação Infantil (Dissertação de mestrado). Faculdade de Educação, Universidade do Estado do Rio de Janeiro, Rio de Janeiro, Brasil.

Souza, Y. C. (2009). Atravessando a Linha Vermelha Programa "Nova Baixada" de Educação Infantil - Discutindo a diversidade étnico-racial e cultural na formação docente (Dissertação de mestrado). Pós-Graduação em Educação, Universidade do Estado do Rio de Janeiro, Duque de Caxias, Brasil.

Souza, R. T. de. (2019). Políticas Públicas de Educação Infantil no municipio de Itaborai/RJ: ampliação e acesso a creche (Dissertação de mestrado). Universidade do Estado do Rio de Janeiro, 2019.

Soares, M. (1989). Alfabetização no Brasil - O Estado do conhecimento. Brasília: INEP/MEC.

Soares, M., Maciel, F. (2000). Alfabetização - Série Estado do Conhecimento. Brasilia: MEC/INEP. 
Sposito, M. P. (2009). O Estado da Arte sobre Juventude na Pós-Graduação Brasileira: Educação, Ciências Sociais e Serviço Social (1999-2006). Belo Horizonte, MG: Argvmentvm.

Sposito, M. P. (2009). Os jovens na Pós-Graduação: um balanço da produção discente em Educação, Serviço Social e Ciências Sociais (1999-2006)", In Sposito, M. P., O Estado da Arte sobre Juventude na Pós-Graduação Brasileira: Educação, Ciências Sociais e Serviço Social (1999-2006) (Vol. 1, pp. 17-56). Belo Horizonte, MG: Argvmentvm.

Torres, L. L., Palhares, J. A. (2014). Metodologia de investigação em Ciências Sociais da Educação. Instituto de Educação da Universidade do Minho, Minho: Edições Húmus.

Vasconcellos, V. M. R. (2008). Ordenamento Legal para a educação infantil e os desafios para os gestores municipais. In Vasconcellos, T. (Org.), Reflexões sobre infância e cultura. Niterói: EDUFF.

Zadminas, M. R. (2016). PEl egressos da UERJ no facebook: uma busca pelas falas a respeito dos saberes e prática no berçário carioca (Dissertação de mestrado). Faculdade de Educação, Universidade do Estado do Rio de Janeiro, Rio de Janeiro, Brasil.

\section{Anne Patricia Pimentel Nascimento da Silva}

Mestre em Educação pelo Programa de Pós-Graduação em Educação da Universidade do Estado do Rio de Janeiro (UERJ), no Rio de Janeiro, RJ, Brasil; professora da rede municipal de Educação do Rio de Janeiro. Psicopedagoga no Núcleo de Apoio Psicopedagógico (NAPp/ UNISUAM) e tutora a distância de Pedagogia da UERJ/ Consórcio CEDERJ.

\section{Roberta Teixeira de Souza}

Mestre em Educação pelo Programa de Pós-graduação em Educação da Universidade do Estado do Rio de Janeiro (UERJ), no Rio de Janeiro, RJ, Brasil; membro do Núcleo de Estudos da Infância: Pesquisa e Extensão (NEI:P\&E/UERJ). Professora da Secretaria estadual de Educação do Rio de Janeiro (SEEDUC/RJ). Coordenadora da Educação Infantil na Secretaria Municipal de Educação, Cultura e Turismo de Itaborai/RJ.

\section{Vera Maria Ramos de Vasconcellos}

Doutora em Psicologia do Desenvolvimento \& Social pela University of Sussex, UK; mestre em Psicologia Escolar pela Pontifícia Universidade Católica do Rio de Janeiro (PUC-RIO), no Rio de Janeiro, RJ, Brasil; professora aposentada da Universidade Federal Fluminense (1977 - 2002, Titular desde 1994) e professora titular aposentada da Universidade do Estado do Rio de Janeiro (2002 - 2019), membro do Programa de Pós-Graduação em Educação (Proped/UERJ).

\section{Endereço para correspondência}

Anne Patricia Pimentel Nascimento da Silva; Roberta Teixeira de Souza;

Vera Maria Ramos de Vasconcellos

Universidade do Estado do Rio de Janeiro

Programa de Pós-Graduação em Educação (Proped/ UERJ)

Rua São Francisco Xavier, 524, $12^{\circ}$ andar, sala 13037 - F Maracanã, 20550900

Rio de Janeiro, RJ, Brasil 